\title{
Robotic left ventral segmentectomy
}

Giuseppe Mangiameli, MD, $\mathrm{PhD},{ }^{\mathrm{a}, \mathrm{b}}$ and Marion Durand, $\mathrm{MD}, \mathrm{PhD},{ }^{\mathrm{c}}$ Milan, Italy, and Antony, France

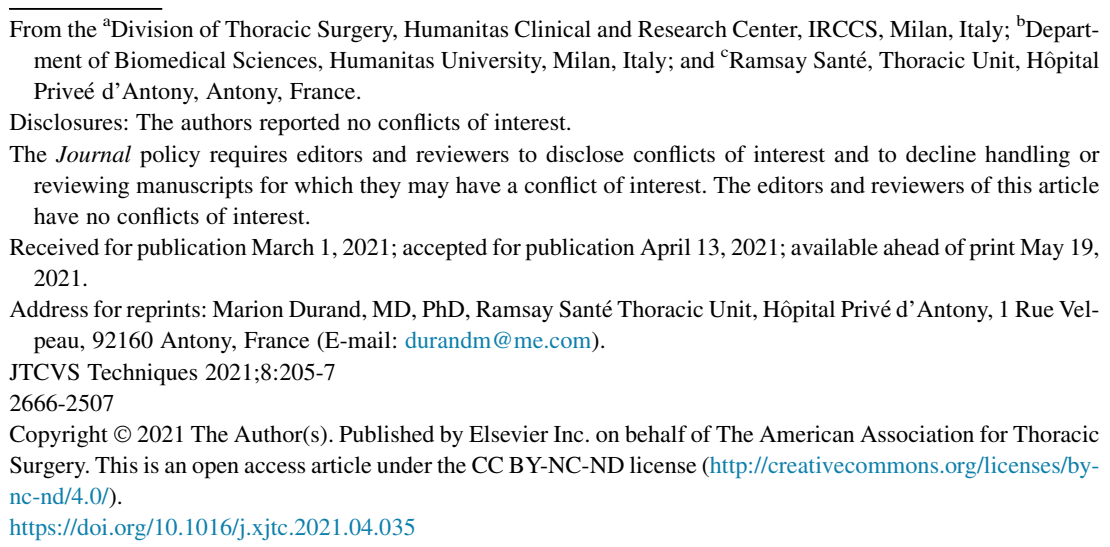

Video clip is available online.

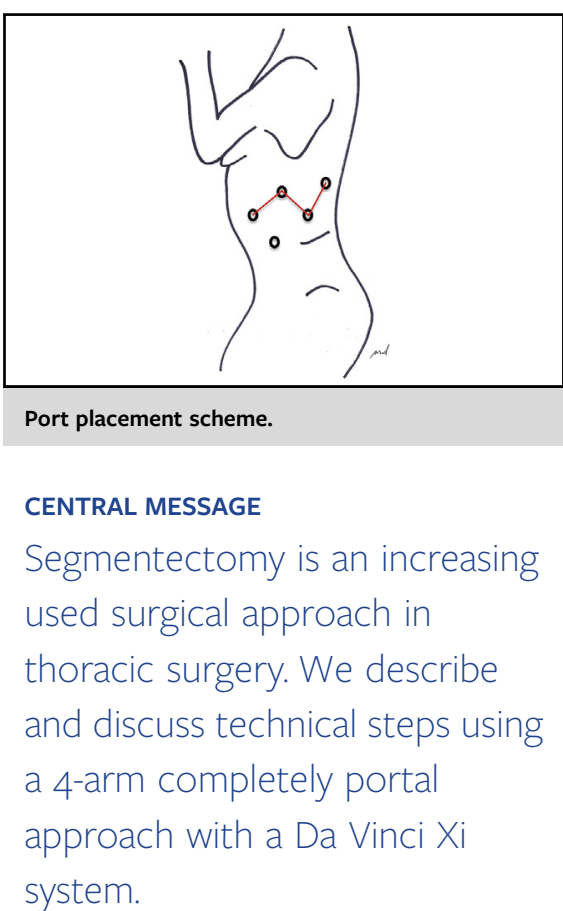

A recurrent lingula artery had to be ligated to avoid its tear during later fissure stapling. Subsequently, anterior exposure was conducted. This allowed the completion of node harvest (zone 5, 12, and 13) and S3 vein, artery, and bronchus control. In a sequence, vein 3 and artery 3 were controlled and manually ligated (silk 0 size, $10 \mathrm{~cm}$ length). Bronchus 3 was mechanically stapled through the port access $(8.5 \mathrm{~mm}$ Echelon Powered $35-\mathrm{mm}$ white load, Ethicon, Somerville, NJ). S3 lung mapping was completed 20 seconds after ICG intravenous injection $(20 \mathrm{mg}$; ie, $8 \mathrm{~mL}$ followed by $10 \mathrm{~mL}$ saline flush). Both intersegmental plans stapling required $560-\mathrm{mm}$ green loads from the right-hand port (12 mm Echelon Powered Flex). Airtight patency was checked under water, specimen removed in a bag through the port access hole, and chest closed with a $24 \mathrm{~F}$ chest tube through the anterior port.

Postoperative course was uneventful. Chest tube was removed on postoperative day 1 and the patient was discharged on day 3. Final pathology confirmed pT2 N0 R0 squamous cell carcinoma. Institutional review board approval was not required for the publication of the study data, and the patient's informed consent was received.

\section{COMMENTS}

Segmentectomy is a increasingly used surgical approach in thoracic surgery because it combines en bloc resection the artery and zone 11, 12 node harvest were performed. 


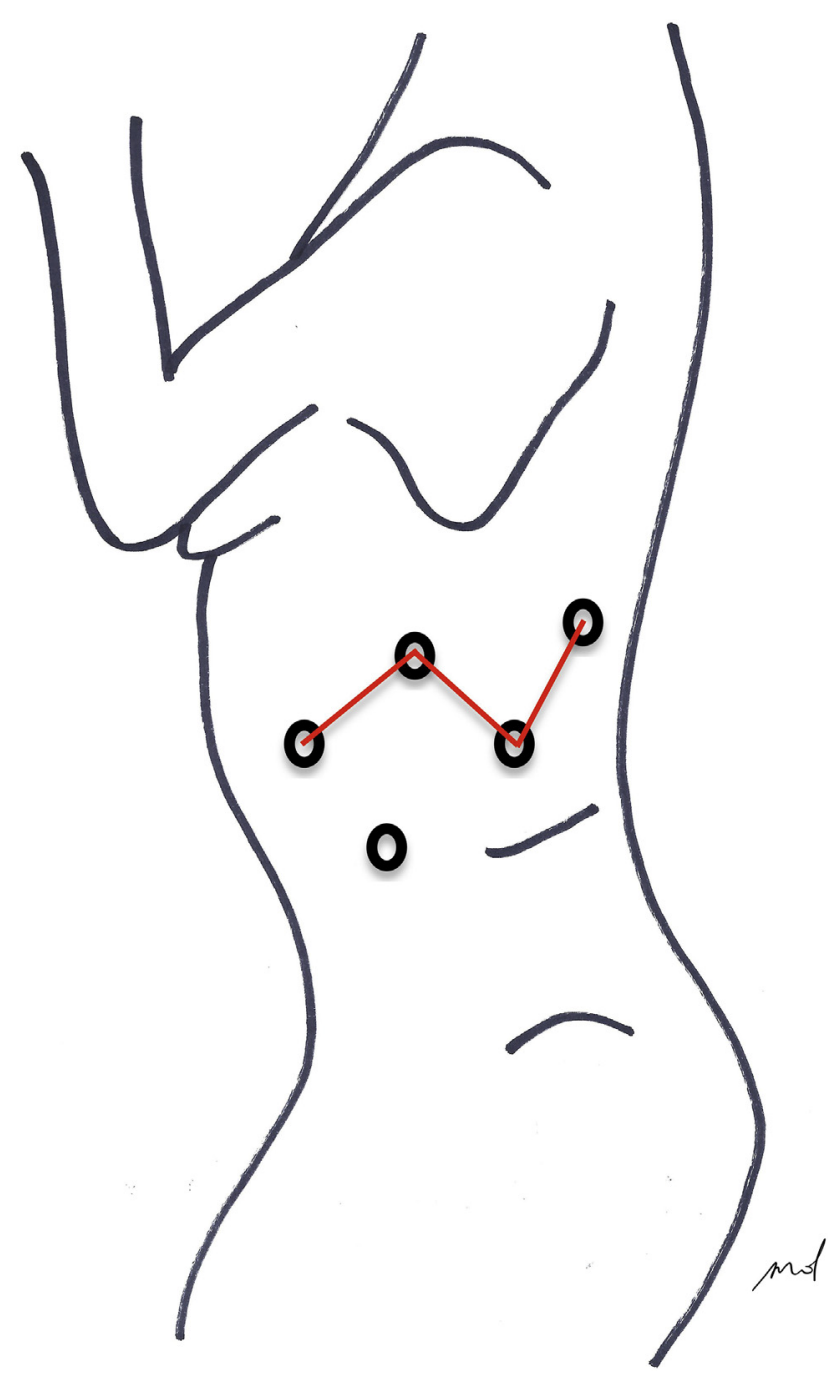

FIGURE 1. Port placement scheme.

with radical node harvest and lung sparing strategy. ${ }^{4}$ It is admitted for a $<2 \mathrm{~cm}$ lesion but will also be considered for larger tumors in comorbid patients as a best-care strategy versus radiotherapy. ${ }^{4}$ Culmenectomy was considered due to the large size of the tumor $(40 \mathrm{~mm})$, but a 8 Charlson index score and a strictly centred S3 location in the emphysematous zone led us to prefer a segmentectomy.

Complex anatomical segmentectomy can be very challenging using traditional video-assisted thoracoscopic surgery or even open surgery (ie, sizing of structure, versatility of exposure, and lung mapping). As of today, we failed to find a technical description for such a procedure. S3 segmentectomy can be tricky, especially on the left side as upper lobe vascularization might be complex and delicate to control. The robotic system offers significant technical skills and vision that ensure its achievement as demonstrated in Video 1.

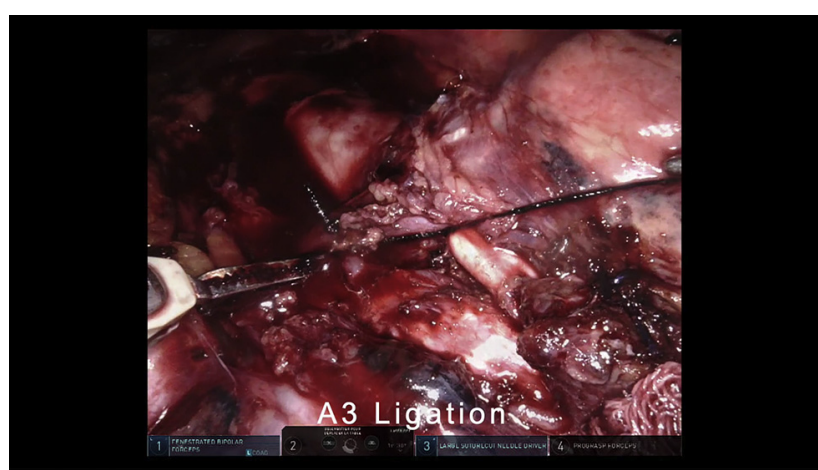

VIDEO 1. Four-arm robotic left S3 segmentectomy. Video available at: https://www.jtcvs.org/article/S2666-2507(21)00376-X/fulltext.

Several technique-specific characteristics can be summarized:

- Trocar placement The partial W port setting allows $30^{\circ}$ camera visual control of the whole chest cavity sparing tools conflict. ${ }^{5}$ A distant/lower port access offers the assistant a larger movement range (Figure 1).

- Reproducibility In our experience this positioning is the same for any procedure.

- Utility incision A 15-mm port ensures airtight patency and affords a low-pressure capnothorax $(5 \mathrm{~mm} \mathrm{Hg})$.

- Robotic stapling sparing Sparing is not only a cost issue. Robotic stapling requires 212 -mm ports, considering that segmentectomies need both anterior and posterior axes of stapling. Posterior port might be painful especially for a patient with smaller intercostal spaces. The more distal the dissection, the more manual targeting and ligation of vessels are required. Manual vessel ligation is also a safety key point and skills concern; the versatility of hand-wrist instruments allows to maintain the vessel's axis as well as ensure routine surgeon hard skills practice.

- ICG We use a $8 \mathrm{~mL}(20 \mathrm{mg}$ ) dose for each patient, whatever weight and sex, waiting no more than 20 seconds before marking to avoid product spreading. We also routinely check the partial wash out of ICG due to cardiac output to be sure of the venous patency on the remaining segments.

\section{CONCLUSIONS}

Robotic hand-wrist surgery offers optimal technical conditions for a safe and rigorous vessel control and node harvest during a complex segmentectomy.

\section{References}

1. Durand M. Four arm robotic double right segmentectomies S3 \& S7-10 with fluorescence lung mapping. J Vis Surg. 2020;6:40.

2. Durand M. Four-arm robotic sleeve right upper lobectomy. Ann Cardiothorac Surg. 2019;8:286-7.

3. Novellis P, Bottoni E, Alloisio M, Velez-Cubian FO, Toloza EM, Veronesi G. Robotic-assisted pulmonary segmentectomies. J Vis Surg. 2018;4:166. 
4. Cao C, Chandrakumar D, Gupta S, Yan TD, Tian DH. Could less be more? A systematic review and meta-analysis of sublobar resections versus lobectomy for non-small cell lung cancer according to patient selection. Lung Cancer. 2015; 89:121-32.
5. Durand M, Dabboura E, Lamonerie L, Herkert A, Zarka V, Carrier AS, et al. Four-arm robotic lung resection versus muscle-sparing mini-thoracotomy: retrospective experience. J Thorac Dis. 2019;11: 1433-42. 